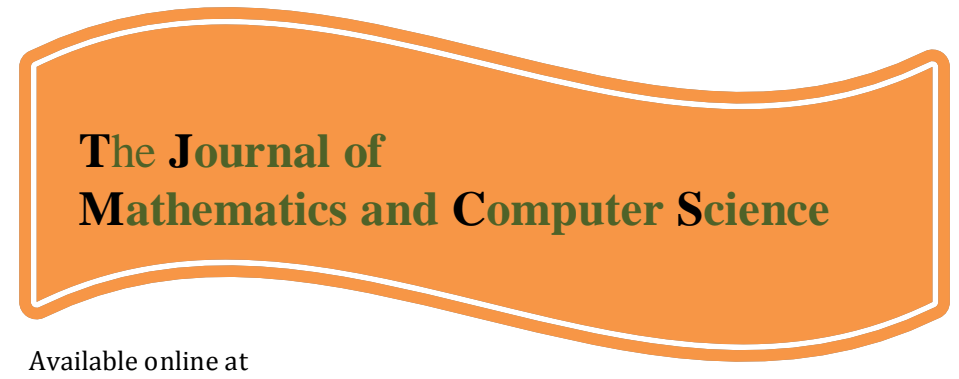

http://www.TJMCS.com

The Journal of Mathematics and Computer Science Vol .2 No.2 (2011) 329-336

\title{
Representing the New Model for Improving K-Means Clustering Algorithm based on Genetic Algorithm
}

\author{
Rouhollah Maghsoudi1, ${ }^{*}$, Arash Ghorbannia Delavar², Somayye Hoseyny3, Rahmatollah \\ Asgari $^{4}$, Yaghub Heidari 5 \\ Department of Computer, Nour Branch, Islamic Azad University, Nour, Iran \\ Payame Noor University, Tehran, Iran, a_ghorbannia@pnu.ac.ir \\ Payame Noor University of Shahrerey, sce.hoseyny@gmail.com \\ Islamic Azad University of Semnan, r_askaree@yahoo.com \\ Department of Electrical, Nour Branch, Islamic Azad University, Nour, Iran
}

Received: September 2010, Revised: December 2010

Online Publication: January 2011

\begin{abstract}
Data clustering into appropriate classes and categories is one of the important topic in pattern recognition. It is very good and very efficient that the number of data which misclassified is minimized or in other words data that classified in each class has been possible as much possible similarity together. In this article at the first, a fundamental method of data clustering which named K-Means Clustering was expressed and then with genetic algorithm, our proposal model that we named it GA-Clustering for improving K-Means method has been introduced. Finally, the said model was examined on some of the well-known data set. Results show that our method clusters data better than traditional K-Means Clustering algorithm significantly.
\end{abstract}

\footnotetext{
1,* Corresponding author:

E-mail address: rcemaghsoudy@yahoo.com (Phone: +98 122625 4590)

2 Payame Noor University, Tehran, Iran

${ }^{3}$ Computer Department of Payame Noor University of Shahrerey

${ }^{4}$ Mechanical Department of Islamic Azad University of Semnan

${ }^{5}$ Electrical Depertment of Islamic Azad University of Nour (Phone: +98 122625 4590)
} 
Keywords: Pattern Recognition, Clustering, K-Means, Genetic Algorithm.

\section{Introduction}

At the first it's necessary to explain about clustering. This method is an instrument for unsupervised classification of data sets. In fact, clustering is an unsupervised separating technique that get data as vectors in multi-dimensional search space and then put them in categories or clusters. With this assumption that patterns of a cluster have a similarity mean while the patterns of different clusters don't similar together. Therefore we must find a similarity measurement for some of data such that these patterns set in an identical cluster. One similarity measurement can be euqlidician distance of $\mathrm{X}$ and $\mathrm{Y}$ patterns that defined as below:

$\mathrm{D}=\|\mathrm{x}-\mathrm{z}\|$

If this distance becomes fewer then similarity degree will be superior.

Clustering in a N-dimensional search space is same as dividing process of $\mathrm{N}$-point set into $\mathrm{K}$ subset or subspace (cluster or category) accordingly similarity or unsimilarity degree of data. Let's go show these $N$ points $\{x 1, x 2, \ldots, x n\}$ become as $S$ that it includes $K$ clusters $C_{1}, C_{2}, \ldots, C_{k}$, then

$\mathrm{C}_{\mathrm{i}} \neq \mathbf{0} \quad$ for $\mathrm{i}=\mathbf{1}, \ldots, \mathrm{k}$,

$C_{i} \cap C_{j}=0$ for $i=1, \ldots, k, j=1, \ldots, k$ and $i \neq j$

And $\cup \mathrm{k}_{\mathrm{i}=1} \mathrm{Ci}=\mathrm{s}$

In this paper K-Means clustering method will be discussed firstly.

\section{K-Means Algorithm}

As mentioned above, this method is one of the clustering techniques that represented by Mc. Queen in 1967. K-Means can be summarized in some steps totally:

Step 1: we select $K$ points as cluster centers $\mathrm{Z}_{1}, \mathrm{Z}_{2}, \mathrm{Z}_{3}, \ldots, \mathrm{Z}_{\mathrm{k}}$ among $\mathrm{N}$ data points randomly.

Step 2: we allocate $X_{i}, i=1,2, \ldots, n$ to hypothetical cluster $C_{j}$ if and only if

$\left\|x_{i}-z_{j}\right\|<\left\|x_{i}-z_{p}\right\|, P=1,2, \ldots, K$, and $j \neq p .-$

Step 3: the new centers of clusters $\mathrm{Z}_{1}, \mathrm{Z}_{2}, \mathrm{Z}_{3}, \ldots, \mathrm{Z}_{\mathrm{k}}$ are calculated as below:

$\mathrm{Z}_{\mathrm{i}}{ }^{*}=1 / \mathrm{n}_{\mathrm{i}} \quad \Sigma \mathrm{X}_{\mathrm{j}}, \mathrm{i}=1,2, \ldots, \mathrm{k}$

$\mathrm{x}_{\mathrm{j}} \in \mathrm{c}_{\mathrm{i}}$

That in is the elements which belong to cluster $\mathrm{C}_{j}$.

Step 4: if $\mathrm{z}_{\mathrm{i}}=\mathrm{z}_{\mathrm{i}}, \mathrm{i}=1,2, \ldots, \mathrm{K}$ then algorithm finishes, otherwise continue from step 2.

In conditions that process doesn't terminate in step4 naturally, it's better algorithm repeat as maximum predefined iterations.

Expressed statements was summation of K-Means clustering algorithm. But next method or it's better to say our method is clustering process using genetic algorithms. Of course our technique is composition of K-Means and GA together.

\section{Genetic Algorithm}

Genetic algorithms are improvement techniques and some methods of accident at searching who are produced from concepts of natural selection recommendation and evolutional trends. This Darwin's evolutional recommendation is so display, in nature who can believe that has more competence for life and others will die in competition. Indeed, genetic algorithms are resolution systems base of computer and they use calculation models in some basic elements of gradual evolution in design and performance. 
General element on genetic algorithms is applying an accidental searching instead of a certain and defined algorithm. Genetic algorithm is a member of more extensive family so called evolutional algorithms.

Extensive groups in evolutional algorithms are :

*Genetic algorithms

*Evolutional programming

*Evolutional strategies

*Classification systems

*Genetic programming

All of them are identical in a basic meaning and simulation of evolution has done with selection. Mutation and reproduction trends. Genetic algorithms have structures that conclude with selection rules and other process.

Any one in valuational population receives fitness from environment. Reproduction is happen on individual who has more fitness. Mutation and crossover are practice on individuals and change them with a revelatory dependent.

General structure of a genetic algorithm is defined:

\subsection{A seudo-code for Genetic Algorithm}

In this part we display a general semi-code for genetic algorithms and their processes.

In this section we try to give an absolutely view of base and processes of genetic algorithms. Later we describe this process generally specific semi-code for genetic algorithms is:

1. Representing and encoding: Defines a genetic show from specific system.

2. Start: Produces an accidental population containing n chromosome.

3. Fitness: Assesses fitness of each chromosome in population and selects among them more value able chromosome so call particular initiative population for genetic algorithm.

4. New population: Apply some processes in following way to create new population:

4.1 Selection: Selects 2 chromosomes base of their fitness in population.

4.2 Crossover: Cross these 2 certain chromosomes and produces new children and adds them to the new population.

4.3 Mutation: With a mutation probability, it changes parent chromo some series and adds this mutant chromo some to the new population.

4.4 Accepting: Fits these new chromosomes and selects them if they have move competence than initiative population; if they are not deletes them from new chromosome lists.

Duty of GA is finding proper cluster centers $Z_{1}, Z_{2}, Z_{3}, \ldots, Z_{k}$ in such away that clustering standard of M can be minimize. 
5. Replace: in this phase the new population chromosomes are replaced which chromosomes of old population that have fewer values rather than them and current population is known as primary population.

6. Test: if termination condition is satisfied then algorithm stops and selects best solution among population.

7. Loop: jump to step 4.

If we want to display a flowchart of genetic algorithm, will be as you see in below:

- Representation and encoding of problem

- Start

- Fitness

- Creation of new population

- Selection

- Crossover

- Mutation

- Accepting

- Replace

- Test

- Loop

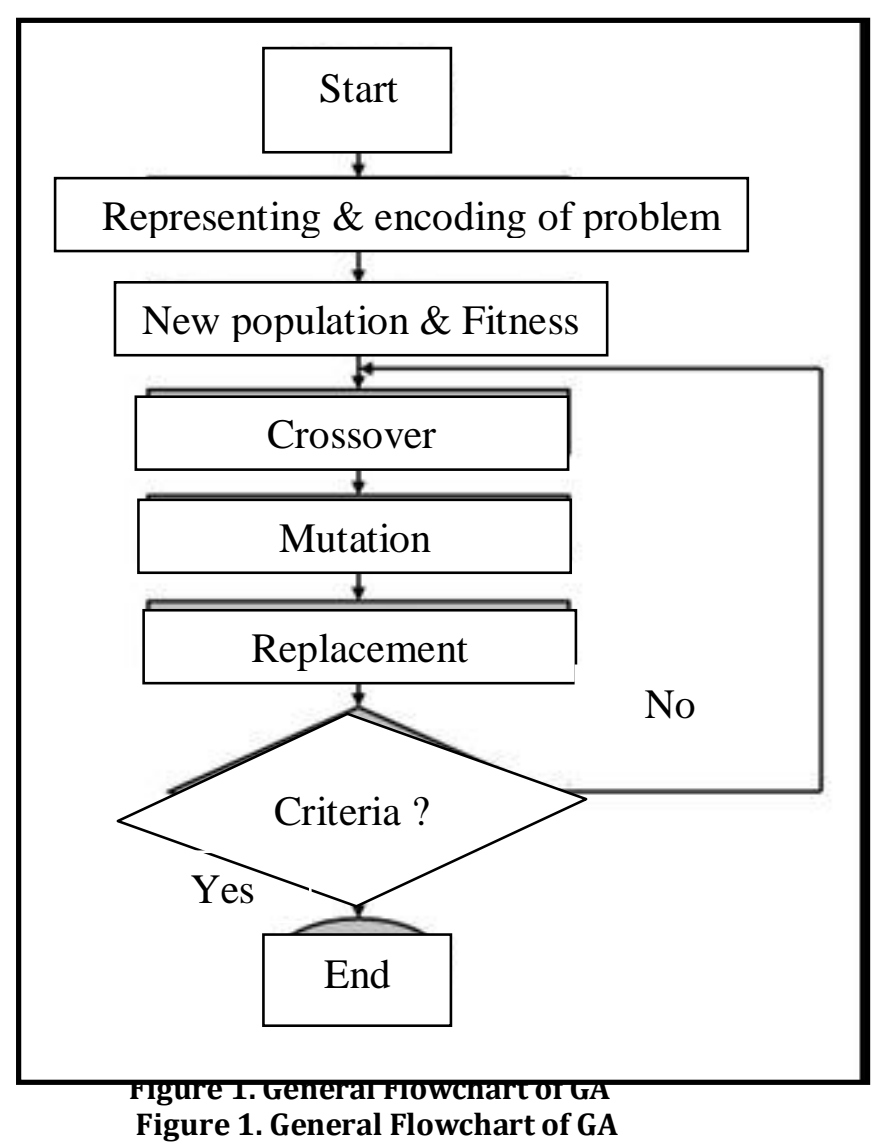




\section{Clustering using GA}

In clustering using GA defines a measurement naming $\mu$ as below:

$\mu\left(\mathrm{C}_{1}, \mathrm{C}_{2}, \ldots, \mathrm{C}_{\mathrm{k}}\right)=\sum \sum\left\|\mathrm{X}_{\mathrm{j}}-\mathrm{Z}_{\mathrm{i}}\right\|$

Duty of GA is finding proper cluster centers $Z_{1}, Z_{2}, Z_{3}, \ldots, Z_{k}$ in such away that clustering standard of $\mathrm{M}$ can be minimize.

\subsection{Displaying series}

Each series is a collection of real numbers that show K cluster centers. In $\mathrm{N}-\mathrm{D}$ environment, length of each chromosome is identify wit $\mathrm{N}^{*} \mathrm{~K}$.

$$
\left(\begin{array}{lll}
N_{1} & N_{2} & N_{k}
\end{array}\right)
$$

Center of $c_{1} \quad$ center of $c_{2} \quad$ center of $c_{k}$

For example, assume that $\mathrm{N}=4, \mathrm{~K}=3$ (Iris) , this means space of question is $4 \mathrm{D}$ and viewable clusters are 3. So chromosomes are in this form:

$\underline{1.5000 \quad 3.5000 \quad 5.2000 \quad 0.4000}$

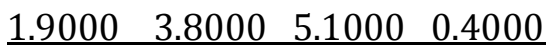

$\begin{array}{llll}1.6000 & 3.4000 & 5.0000 & 0,2491\end{array}$

That showing 3 cluster centers,

$\underline{0.4000 \quad 5.1000 \quad 3.8000 \quad 1.9000}$

$\underline{1.5000 \quad 3.5000 \quad 5.2000 \quad 0.4000}$

$\underline{0.2491 \quad 5.0000 \quad 3.4000 \quad 1.6000}$

Each of these real numbers are an indivisible genes.

\subsection{Population initialization}

K cluster centers are accidentally selected from available list and insert in one chromosome. This trend repeats for all $\mathrm{P}$ chromosomes producing population. Indeed $\mathrm{P}$ is population size. 


\subsection{Fitness computation}

Access of fitness contains two processes. In first process, clusters, base of centers is chromosomes, will produce. This means any point $X_{i}$ specify to one of the clusters $C_{j}$ with center of $Z_{j}$, if:

$$
\left\|X_{i-} Z_{j}\right\|<\left\|X_{i}-Z_{p}\right\|, \mathrm{P}=1,2, \ldots, \mathrm{K} \text { and } \mathrm{j} \neq \mathrm{P}
$$

After clustering is done, available cluster centers in chromosomes replace with average of any cluster's points. For cluster $C_{i}$, new center $Z_{i}$ accesses in this way:

$Z_{i}^{*}=\frac{1}{n_{i}} \sum X_{i} \quad \mathrm{i}=1,2, \ldots, \mathrm{K}$

For define this subject. We give an example. Assume that the first cluster center was ( 51.6 , 72.3 ). After clustering, there are 2 other points in this space:

$(52.0,74.0),(50.0,70.0)$.So because this new center point of cluster 1 is identical with $(10.0+74.0+7203) / 3,(50.0+52.0+51.6) / 3$, this new center $(51.2,72.1)$ replace with previous center $(51.6,72.3)$ in relevant chromosome.

The clustering standard or $\square$ is account in this way:

$\mu=\sum_{I=1}^{k} \mu_{i} \quad \mu_{I}=\sum\left\|X_{j}-Z_{j}\right\|$

Also fitness dependent accesses in this way:

$\mathrm{F}=\frac{1}{\mu}$

\subsection{Useable genetic operators}

Crossover: Crossover is a probability trend that we change information among 2 chromosome called parent in order to produce 2 off springs. In this paper we apply one-point crossover with fixed crossover value $P_{c}=0.8$.

Mutation: Each chromosome participates in mutation with a fixed probability. Supernatant decimal is apply for chromosomes. To mutation we use this way. A number of $\delta$ accidentally produces with monotonous distribution in span [0.1]. If the number of mutant gene is $V$, there is:

$\mathrm{V} \pm 2 * \delta^{*} \mathrm{~V}, \quad \mathrm{~V} \neq 0$

$\mathrm{V} \pm 2 * \delta \quad, \mathrm{V}=0$

Positions of - and + happened with similar probability. For simplify in this project, mutation is done in the form of $\mathrm{V} \pm \delta^{*} \mathrm{~V}$.

\subsection{Stopping criteria}

In this work, calculation trend of fitness, crossover, mutation is done in amount of maximum repeated number. 


\section{The result of simulation and comparison of algorithms}

Before draw a conclusion, we give some definition for kind of parameters. As previously said, the object was assessment of K-means clustering method and proposal trend of GAclustering and comparison of their result. For this, we use some collections of different parameters. The example is Iris Data set. That shows different clusters of a flower and contains 4 feature of Petal's length, Petal's width, Sepal's length, Sepal's width in $\mathrm{cm}$. The parameters are describe in 3 class: $1,0.5,0$.

The number of parameters should be identical with 3. Other parameter collection is called Vowel that contains 871 record parameter. Each record parameter constitutes 3 index $f_{1}, f_{2}, f_{3}$. This parameter should be insert in 6 class. Third parameter collection is called Crude oil that contains 56 record parameters and each record constitutes 5 index. This parameter should insert in 3 class.

GA-clustering implementation is done with these parameters:

Crossover rate (PC): 0.8

Mutation rate (PM): 0.001

Population size (pop-size): 100

Maximum iteration: 100

K-means clustering is done with these parameters:

Number of clusters or K: That there are different parameters in each kind.

Maximum iteration: 100

\section{Observed results}

The numbers in this table are clustering standard or $\mu$; and the lower one can give better conclusion. This conclusion implement more and more and accept with different initiative population on different parameters collections.

Table 1-5: obtained $\mu$ from running two algorithms on Iris data set $(\mathrm{k}=3)$

\begin{tabular}{|c|c|c|}
\hline GA-Clustering & K-Means Clustering & Running times \\
\hline 30.1203 & 32.1988 & 1 \\
\hline 29.6173 & 30.4915 & 2 \\
\hline 29.9909 & 30.7811 & 3 \\
\hline 29.6173 & 30.5039 & 4 \\
\hline
\end{tabular}

Table 1-6: obtained $\mu$ from running two algorithms on Crude oil data set $(k=3)$

\begin{tabular}{|c|c|c|}
\hline GA-Clustering & K-Means Clustering & Running times \\
\hline 278.965148 & 279.743216 & 1 \\
\hline 278.965148 & 279.743216 & 2 \\
\hline
\end{tabular}




\begin{tabular}{|l|l|l|}
\hline 278.965148 & 279.484810 & 3 \\
\hline 278.965148 & 279.597091 & 4 \\
\hline
\end{tabular}

Table 1-7: obtained $\mu$ from running two algorithms on Vowel data set $(k=6)$

\begin{tabular}{|c|c|c|}
\hline GA-Clustering & K-Means Clustering & Running times \\
\hline 149346.489128 & 157460.164831 & 1 \\
\hline 149406.751288 & 149394.803983 & 2 \\
\hline 149346.152169 & 161094.118096 & 3 \\
\hline 149355.823103 & 149373.097180 & 4 \\
\hline
\end{tabular}

\section{Conclusion}

In this paper K-Means algorithm that is one of the popular clustering techniques has been surveyed and tried to apply one of the optimization method named genetic algorithm improve in unsupervised classification procedure. Genetic algorithms are population based methods that use from operators for processing of population chromosomes. In this research, we defined a representation of chromosome string and combine K-Means and GA together. Observing simulations in different runnings show that K-Means clustering based on Genetic algorithm improved clustering measurement $\mu$ better and more efficient rather than pure K-Means considerably.

\section{References:}

[1] Duda R. 0., Hart P. E., and Stark D. G.,Pattern classification, second edition,John Wiley,2000.

[2] Melanie Mitchell, An Introduction to Genetic Algorithms, MIT Press, Cambridge, MA, 2002; first edition, 1996.

[3] Hu Shu-chiung, Genetic algorithm-based clustering technique, Ujjwal Maulik, Sanghamitra Bandyopadhyay, 2004.

[4] Jun Zhang, Member, IEEE, Henry Shu-Hung Chung, Senior Member, IEEE, and Wai-Lun Lo, Member, IEEE, Clustering-based Adaptive Crossover And Mutation Probabilities for Genetic Algorithms, 2006.

[5] Licheng Jiao, Senior Member, IEEE, Jing Liu, and Weicai Zhong, An Organizational Coevolutionary Algorithm for Classification, 2006. 